\title{
Extensible Proof-Producing Compilation
}

\author{
Magnus O. Myreen ${ }^{1}$, Konrad Slind ${ }^{2}$, and Michael J.C. Gordon ${ }^{1}$ \\ ${ }^{1}$ Computer Laboratory, University of Cambridge, Cambridge, UK \\ ${ }^{2}$ School of Computing, University of Utah, Salt Lake City, USA
}

\begin{abstract}
This paper presents a compiler which produces machine code from functions defined in the logic of a theorem prover, and at the same time proves that the generated code executes the source functions. Unlike previously published work on proof-producing compilation from a theorem prover, our compiler provides broad support for user-defined extensions, targets multiple carefully modelled commercial machine languages, and does not require termination proofs for input functions. As a case study, the compiler is used to construct verified interpreters for a small LISP-like language. The compiler has been implemented in the HOL4 theorem prover.
\end{abstract}

\section{Introduction}

Compilers pose a problem for program verification: if a high-level program is proved correct, then the compiler's transformation must be trusted in order for the proof to carry over to a guarantee about the generated executable code. In practice there is also another problem: most source languages (C, Java, Haskell etc.) do not have a formal semantics, and it is therefore hard to formally state and verify properties of programs written in these languages.

This paper explores an approach to compilation aimed at supporting program verification. We describe a compiler which takes as input functions expressed in the logic of a theorem prover, compiles the functions to machine code (ARM, $\mathrm{x} 86$ or PowerPC) and also proves that the generated code executes the supplied functions. For example, given function $f$ as input

$$
f\left(r_{1}\right)=\text { if } r_{1}<10 \text { then } r_{1} \text { else let } r_{1}=r_{1}-10 \text { in } f\left(r_{1}\right)
$$

the compiler can generate ARM machine code

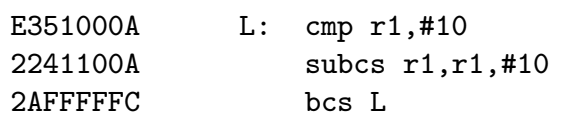

and automatically prove a theorem which certifies that the generated code executes $f$. The following theorem states, if register one (r1) initially holds value $r_{1}$, then the code will leave register one holding value $f\left(r_{1}\right)$. The theorem is expressed as a machine-code Hoare triple [17] where the separating conjunction ' $*$ ' can informally be read as 'and'.

$$
\left\{\mathrm{r} 1 r_{1} * \mathrm{pc} p * \mathrm{~s}\right\} \quad p: \mathrm{E} 351000 \mathrm{~A}, 2241100 \mathrm{~A}, 2 \mathrm{AFFFFFC}\left\{\mathrm{r} 1 f\left(r_{1}\right) * \mathrm{pc}(p+12) * \mathrm{~s}\right\}
$$


The fact that $f$ is expressed as a function in the native language of a theorem prover means that it has a precise semantics and that one can prove properties about $f$, e.g. one can prove that $f(x)=x \bmod 10$ (here mod is modulus over unsigned machine words). Properties proved for $f$ carry over to guarantees about the generated machine code via the certificate proved by the compiler. For example, one can rewrite the theorem from above to state that the ARM code calculates $r_{1} \bmod 10$ :

$\left\{\mathrm{r} 1 r_{1} * \mathrm{pc} p * \mathrm{~s}\right\} \quad p: \mathrm{E} 351000 \mathrm{~A}, 2241100 \mathrm{~A}, 2 \mathrm{AFFFFFC} \quad\left\{\mathrm{r} 1\left(r_{1} \bmod 10\right) * \mathrm{pc}(p+12) * \mathrm{~s}\right\}$

Proof-producing compilation from a theorem prover has been explored before by many, as will be discussed in Section 6. The contributions that distinguish the work presented here are that the compiler:

1. targets multiple, carefully modelled, commercial machine languages (namely ARM, PowerPC and x86, as modelled by Fox [7, Leroy [1] and Sarkar [6]);

2 . does not require the user to prove termination of the input functions (a restriction posed by the theorem prover in similar work by Li et al. [121314]);

3. can, without any added complexity to the certification proof, handle a range of optimising transformations (Section 4); and

4. supports significant user-defined extensions to its input language (Section 3.1); extensions which made it possible to compile interpreters for a small LISP-like language as a case study (Section [5).

The compilen 1 uses a functional input, which is meant to either be extended directly by the user, as discussed in Section 3.1, or used as a back-end in compilers with more general input languages, e.g. 81314].

This paper builds on the authors' work on post hoc verification of realistically modelled machine code [16 17,18], and certifying compilation 8[12 13 14].

\section{Core Functionality}

The compiler presented in this paper accepts tail-recursive functions as input, functions defined as recursive equations ' $f(\ldots)=\ldots$ ' in a format described in Section 2.1. As output the compiler produces machine code together with a correctness certificate, a theorem which states that the generated machine code executes the function given as input.

The overall compilation algorithm can be broken down into three stages:

1. code generation: generates, without proof, machine code from input $f$;

2. decompilation: derives, via proof, a function $f^{\prime}$ describing themachine code;

3. certification: proves $f=f^{\prime}$.

The remaining subsections describe the input language and code generation that make proving $f=f^{\prime}$ feasible, as well as the mechanism by which $f^{\prime}$ is derived. Section 3 describes extensions to the core algorithm.

${ }^{1}$ The HOL4 source is at http://hol.sf.net/ under HOL/examples/machine-code. 


\subsection{Input Language}

The compiler's input language consists of let-expressions, if-statements and tailrecursion. The language restricts variable names to correspond to names of registers or stack locations.

The following grammar describes the input language. Let $r$ range over register names, $r_{0}, r_{1}, r_{2}$, etc., and $s$ over stack locations, $s_{1}, s_{2}, s_{3}$ etc., $m$ over memory modelling functions (mappings from aligned 32-bit machine words to 32-bit machine words), $f$ over function names, $g$ over names of already compiled functions, and $i_{5}, i_{7}, i_{8}$ and $i_{32}$ over unsigned words of size 5-, 7-, 8- and 32-bits, respectively. Bit-operators \&, ??, !!, «, 》 are and, xor, or, left-shift, right-shift. Operators suffixed with '?' are signed-versions of those without the suffix.

$$
\begin{aligned}
\text { input }::= & f(v, v, \ldots, v)=r h s \\
r h s::= & \text { let } r=\exp \text { in } r h s \\
\mid & \text { let } s=r \text { in } r h s \\
\mid & \text { let } m=m[\text { address } \mapsto r] \text { in } r h s \\
\mid & \text { let }(v, v, \ldots, v)=g(v, v, \ldots, v) \text { in } r h s \\
\mid & \text { if guard then } r h s \text { else } r h s \\
& f(v, v, \ldots, v) \\
\mid & (v, v, \ldots, v) \\
\text { exp }::= & x|\neg x| s\left|i_{32}\right| x \text { binop } x \mid \text { m address }\left|x \ll i_{5}\right| x \gg i_{5} \mid x \gg . i_{5} \\
\text { binop }::= & +|-| \times \mid \text { div }|\&| ? ? \mid ! ! \\
\text { cmp }::= & <|\leq|>|\geq|<.|\leq .|>\text {. }|\geq .|= \\
\text { guard }::= & \text { guard } \mid \text { guard } \wedge \text { guard } \mid \text { guard } \vee \text { guard } \mid x \text { cmp } x \mid x \& x=0 \\
\text { address }::= & r\left|r+i_{7}\right| r-i_{7} \\
x::= & r \mid i_{8} \\
v::= & r|s| m
\end{aligned}
$$

This input language was designed to be machine independent; programs constructed from this grammar can be compiled to any of the target languages: ARM, x86 and PowerPC. However the input language differs for each target in the number of registers available $\left(r_{0} \ldots r_{12}\right.$ for ARM, $r_{0} \ldots r_{6}$ for $\mathrm{x} 86$ and $r_{0} \ldots r_{31}$ for PowerPC) and some detailed restrictions on the use of $x$ and div.

\subsection{Code Generation}

The input language was designed to mimic the operations of machine instructions in order to ease code generation. Each let-expression usually produces a single instruction, e.g.

$$
\begin{array}{lll}
\text { let } r_{3}=r_{3}+r_{2} \text { in } & \text { generates ARM code } & \text { add } r 3, r 3, r 2 \\
\text { let } r_{3}=r_{3}+r_{2} \text { in } & \text { generates x } 86 \text { code } & \text { add ebx,edx } \\
\text { let } r_{3}=r_{3}+r_{2} \text { in } & \text { generates PowerPC code } & \text { add } 3,3,2
\end{array}
$$


In some cases one let-expression is split into a few instructions, e.g.

$$
\begin{array}{ll}
\text { let } r_{3}=r_{0}-r_{2} \text { in } \quad \text { generates } x 86 \text { code } \quad \begin{array}{l}
\text { mov ebx, eax } \\
\text { sub ebx, edx }
\end{array} \\
\text { let } r_{3}=5000 \text { in } \quad \text { generates ARM code } & \begin{array}{l}
\text { mov } r 3, \# 19 \\
\text { mov } r 3, r 3,1 s 18 \\
\end{array} \quad \text { add } r 3, r 3, \# 136
\end{array}
$$

The code generator was programmed to use a few assembly tricks, e.g. on x86 certain instances of addition, which would normally require two instructions (mov followed by add), can be implemented as a single load-effective-address lea:

$$
\text { let } r_{3}=r_{0}+r_{2} \text { in generates x } 86 \text { code lea ebx, [eax+edx] }
$$

A combination of compare and branch are used to implement if-statements, e.g.

$$
\text { if } r_{3}=45 \text { then } \ldots \text { else } \ldots \text { generates ARM code } \begin{aligned}
& \text { cmp } r 3, \# 45 \\
& \text { bne L1 }
\end{aligned}
$$

Function returns and function calls generate branch instructions.

The compiler generates a list of assembly instructions, which is translated into machine code using off-the-shelf assemblers: Netwide Assembler nasm [1] for x86 and the GNU Assembler gas [2] for ARM and PowerPC. Note that these tools do not need to be trusted. If incorrect code is generated then the certification phase, which is to prove the correctness certificate, will fail.

\subsection{Proving Correctness Theorem}

The theorem certifying the correctness of the generated machine code is proved by first deriving a function $f^{\prime}$ describing the effect of the generated code, and then proving that $f^{\prime}$ is equal to the original function to be compiled. Function $f^{\prime}$ is derived using proof-producing decompilation [18]. This section will illustrate how decompilation is used for compilation and then explain decompilation.

Example. Given function $f$, which traverses $r_{0}$ steps down a linked-list in $m$,

$$
\begin{aligned}
& f\left(r_{0}, r_{1}, m\right)= \\
& \text { if } r_{0}=0 \text { then }\left(r_{0}, r_{1}, m\right) \text { else } \\
& \text { let } r_{1}=m\left(r_{1}\right) \text { in } \\
& \text { let } r_{0}=r_{0}-1 \text { in } \\
& \quad f\left(r_{0}, r_{1}, m\right)
\end{aligned}
$$

Code generation produces the following x86 code.

$\begin{array}{ll}\text { 0: } & 85 \mathrm{C0} \\ \text { 2: } & 7405 \\ \text { 4: } & 8 \mathrm{B0} 9 \\ \text { 6: } & 48 \\ \text { 7: } & \text { EBF7 }\end{array}$

L1: test eax, eax jz L2 mov ecx, [ecx] dec eax jmp L1 
Proof-producing decompilation is applied to the generated machine code. The decompiler takes machine code as input and produces a function $f^{\prime}$ as output,

$$
\begin{aligned}
& f^{\prime}(e a x, e c x, m)= \\
& \text { if eax \& eax }=0 \text { then }(e a x, e c x, m) \text { else } \\
& \text { let } e c x=m(e c x) \text { in } \\
& \text { let } e a x=e a x-1 \text { in } \\
& f^{\prime}(e a x, e c x, m)
\end{aligned}
$$

together with a theorem (expressed as a machine-code Hoare triple 17 18) which states that $f^{\prime}$ accurately records the update executed by the machine code. The decompiler derives $f^{\prime}$ via proof with respect to a detailed processor model written by Sarkar [6]. Here eip asserts the value of the program counter.

$$
\begin{aligned}
& f_{\text {pre }}^{\prime}(\text { eax }, e c x, m) \Rightarrow \\
& \{(\text { eax }, \text { ecx }, \mathrm{m}) \text { is }(\text { eax }, e c x, m) * \text { eip } p * \mathrm{~s}\} \\
& p: 85 \mathrm{C} 074058 \mathrm{~B} 0948 \mathrm{EBF} 7 \\
& \left\{(\text { eax }, \text { ecx, } \mathrm{m}) \text { is } f^{\prime}(\text { eax }, \text { ec } x, m) * \operatorname{eip}(p+9) * \mathrm{~s}\right\}
\end{aligned}
$$

The decompiler also automatically defines $f_{\text {pre }}^{\prime}$, which is a boolean-valued function that keeps track of necessary conditions for the Hoare triple to be valid as well as side-conditions that are needed to avoid raising hardware exceptions. In this case, $e c x$ is required to be part of the memory segment modelled by function $m$ and the underlying model requires $e c x$ to be word-aligned $(e c x \& 3=0)$, whenever $e a x \& e a x \neq 0$.

$$
\begin{aligned}
& f_{\text {pre }}^{\prime}(\text { eax }, \text { ecx }, m)= \\
& \quad \text { if eax \& eax }=0 \text { then true else } \\
& \quad f_{\text {pre }}^{\prime}(\text { eax }-1, m(e c x), m) \wedge \text { ec } x \in \text { domain } m \wedge(e c x \& 3=0)
\end{aligned}
$$

Next the compiler proves $f=f^{\prime}$. Both $f$ and $f^{\prime}$ are recursive functions; thus proving $f=f^{\prime}$ would normally require an induction. The compiler can avoid an induction since both $f$ and $f^{\prime}$ are defined as instances of tailrec:

$$
\text { tailrec } x=\text { if }(G x) \text { then tailrec }(F x) \text { else }(D x)
$$

The compiler proves $f=f^{\prime}$ by showing that the components of the tailrec instantiation are equal, i.e. for $f$ and $f^{\prime}$, as given above, the compiler only needs to prove the following. ( $f_{\text {pre }}^{\prime}$ is not needed for these proofs.)

$$
\begin{array}{rrrl}
G: & \left(\lambda\left(r_{0}, r_{1}, m\right) \cdot r_{0} \neq 0\right) & =(\lambda(\text { eax }, \text { ecx }, m) \cdot \text { eax \& eax } \neq 0) \\
D: & & \left(\lambda\left(r_{0}, r_{1}, m\right) \cdot\left(r_{0}, r_{1}, m\right)\right) & =(\lambda(\text { eax }, \text { ecx }, m) \cdot(\text { eax }, \text { ecx }, m)) \\
F: & & \left(\lambda\left(r_{0}, r_{1}, m\right) \cdot\left(r_{0}-1, m\left(r_{1}\right), m\right)\right) & =(\lambda(\text { eax }, \text { ecx }, m) \cdot(\text { eax }-1, m(\text { ecx }), m))
\end{array}
$$

The code generation phase is programmed in such a way that the above component proofs will always be proved by an expansion of let-expressions followed by rewriting with a handful of verified rewrite rules that undo assembly tricks, e.g. $\forall w . w \& w=w$. 
The precondition $f_{\text {pre }}^{\prime}$ is not translated, instead $f_{\text {pre }}$ is defined to be $f_{p r e}^{\prime}$. The compiler proves the certificate of correctness by rewriting the output from the decompiler using theorems $f^{\prime}=f$ and $f_{\text {pre }}^{\prime}=f_{\text {pre }}$. The example results in:

$$
\begin{aligned}
& f_{\text {pre }}(\text { eax }, e c x, m) \Rightarrow \\
& \{(\text { eax }, \text { ecx }, \mathrm{m}) \text { is }(\text { eax }, e c x, m) * \text { eip } p * \mathrm{~s}\} \\
& p: 85 \mathrm{C} 074058 \mathrm{~B} 0948 \mathrm{EBF} 7 \\
& \{(\text { eax }, \text { ecx }, \mathrm{m}) \text { is } f(\text { eax }, e c x, m) * \operatorname{eip}(p+9) * \mathrm{~s}\}
\end{aligned}
$$

Decompilation. The proof-producing decompilation, which was used above, is explained in detail in 18 . However, a brief outline will be given here.

Decompilation starts by composing together Hoare triples for machine instructions to produce Hoare triples describing one pass through the code. For the above x86 code, successive compositions collapse Hoare triples of the individual instructions into two triples, one for the case when the conditional branch is taken and one for the case when it is not.

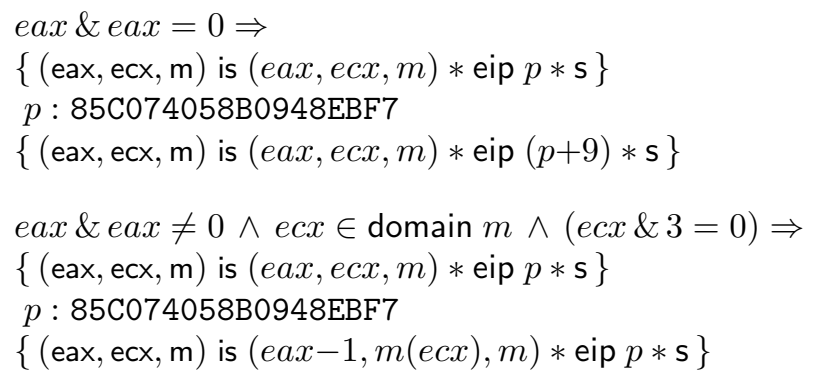

Using these one-pass theorems, the decompiler instantiates the following loop rule to produce function $f^{\prime}$ and the certificate theorem. If $F$ describes a looping pass, and $D$ is a pass that exits the loop, then tailrec $x$ is the result of the loop:

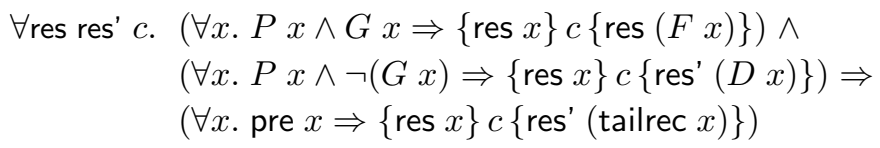

Here pre is the recursive function which records the side-conditions that need to be met (e.g. in this case $P$ is used to record that ecx needs to be aligned).

$$
\text { pre } x=P x \wedge(G x \Rightarrow \text { pre }(F x))
$$

For the above one-pass Hoare triples to fit the loop rule, the decompiler instantiates $G, F, D, P$, res and res' as follows:

$$
\begin{aligned}
& G=\lambda(e a x, e c x, m) .(e a x \& e a x \neq 0) \\
& F=\lambda(e a x, e c x, m) \cdot(e a x-1, m(e c x), m) \\
& D=\lambda(e a x, e c x, m) .(e a x, e c x, m) \\
& P=\lambda(e a x, e c x, m) .(e a x \& e a x \neq 0) \Rightarrow e c x \in \text { domain } m \wedge(e c x \& 3=0) \\
& \text { res }=\lambda(e a x, e c x, m) \text {. (eax, ecx, } \mathrm{m}) \text { is }(e a x, e c x, m) * \text { eip } p * \mathrm{~s} \\
& \text { res } \left.^{\prime}=\lambda(e a x, e c x, m) \text {. (eax, ecx, } \mathrm{m}\right) \text { is }(e a x, e c x, m) * \operatorname{eip}(p+9) * \mathrm{~s}
\end{aligned}
$$




\section{Extensions, Stacks and Subroutines}

The examples above illustrated the algorithm of the compiler based on simple examples involving only registers and direct memory accesses. This section describes how the compiler supports user-defined extensions, stack operations and subroutine calls.

\subsection{User-Defined Extensions}

The compiler has a restrictive input language. User-defined extensions to this input language are thus vital in order to be able to make use of the features specific to each target language.

User-defined extensions to the input language are made possible by the proof method which derives a function $f^{\prime}$ describing the effect of the generated code: function $f^{\prime}$ is constructed by composing together Hoare triples describing parts of the generated code. By default, automatically derived Hoare triples for each individual machine instruction are used. However, the user can instead supply the proof method with alternative Hoare triples in order to build on previously proved theorems.

An example will illustrate how this observation works in practice. Given the following Hoare triple (proved in Section 1) which shows that ARM code has been shown to implement " $r_{1}$ is assigned $r_{1} \bmod 10$ ",

$\left\{\mathrm{r} 1 r_{1} * \mathrm{pc} p * \mathrm{~s}\right\} \quad p: \mathrm{E} 351000 \mathrm{~A}, 2241100 \mathrm{~A}, 2 \mathrm{AFFFFFC} \quad\left\{\mathrm{r} 1\left(r_{1} \bmod 10\right) * \mathrm{pc}(p+12) * \mathrm{~s}\right\}$

the code generator expands its input language for ARM with the following line:

$$
\text { rhs }::=\text { let } r_{1}=r_{1} \bmod 10 \text { in } r h s
$$

Now when a function $f$ is to be compiled which uses this feature,

$$
\begin{aligned}
& f\left(r_{1}, r_{2}, r_{3}\right)= \text { let } r_{1}=r_{1}+r_{2} \text { in } \\
& \text { let } r_{1}=r_{1}+r_{3} \text { in } \\
& \text { let } r_{1}=r_{1} \bmod 10 \text { in } \\
& r_{1}
\end{aligned}
$$

the code generator implements "let $r_{1}=r_{1}$ mod 10 in" using the machine code (underlined below) found inside the Hoare triple. The other instructions are E0811002 for add $r 1, r 1, r 2$ and E0811003 for add $r 1, r 1, r 3$.

\section{E0811002 E0811003 E351000A 2241100A 2AFFFFFC}

The compiler would now normally derive $f^{\prime}$ by composing Hoare triples for the individual machine instructions, but in this case the compiler considers the underlined code as a 'single instruction' whose effect is described by the supplied Hoare triple. It composes the following Hoare triples, in order to derive a Hoare triple for the entire code.

$$
\left\{\mathrm{r} 1 r_{1} * \mathrm{r} 2 r_{2} * \mathrm{pc} p\right\} \quad p: \mathrm{E} 0811002\left\{\mathrm{r} 1\left(r_{1}+r_{2}\right) * \mathrm{r} 2 r_{2} * \mathrm{pc}(p+4)\right\}
$$


$\left\{\mathrm{r} 1 r_{1} * \mathrm{r} 3 r_{3} * \mathrm{pc} p\right\} \quad p: \mathrm{E} 0811003\left\{\mathrm{r} 1\left(r_{1}+r_{3}\right) * \mathrm{r} 3 r_{3} * \mathrm{pc}(p+4)\right\}$

$\left\{\mathrm{r} 1 r_{1} * \mathrm{pc} p * \mathrm{~s}\right\} \quad p: \mathrm{E} 351000 \mathrm{~A}, 2241100 \mathrm{~A}, 2 \mathrm{AFFFFFC} \quad\left\{\mathrm{r} 1\left(r_{1} \bmod 10\right) * \mathrm{pc}(p+12) * \mathrm{~s}\right\}$

The resulting $f^{\prime}$ is trivially equal to $f$ and thus the resulting Hoare triple states that the generated code actually executes $f$.

$$
\begin{aligned}
& \left\{\mathrm{r} 1 r_{1} * \mathrm{r} 2 r_{2} * \mathrm{r} 3 r_{3} * \mathrm{pc} p * \mathrm{~s}\right\} \\
& p: \mathrm{E} 0811002, \mathrm{E} 0811003, \mathrm{E} 351000 \mathrm{~A}, 2241100 \mathrm{~A}, 2 \mathrm{AFFFFC} \\
& \left\{\mathrm{r} 1 f\left(r_{1}, r_{2}, r_{3}\right) * \mathrm{r} 2 r_{2} * \mathrm{r} 3 r_{3} * \mathrm{pc}(p+20) * \mathrm{~s}\right\}
\end{aligned}
$$

It is important to note that the Hoare triples supplied to the compiler need not concern registers or memory locations, instead more abstract Hoare triples can be supplied. For example, in Section [5] the compiler is given Hoare triples that show how basic operations over LISP s-expressions can be performed. The LISP operation car is implemented by ARM instruction E5933000. Here s-expressions are defined as a data-type with type-constructors Dot (pairs), Num (numbers) and Sym (symbols). Details are given in Section 5 .

$$
\begin{aligned}
& \left(\exists x y \cdot v_{1}=\operatorname{Dot} x y\right) \Rightarrow \\
& \left\{\operatorname{lisp}(a, l)\left(v_{1}, v_{2}, v_{3}, v_{4}, v_{5}, v_{6}\right) * \mathrm{pc} p\right\} \\
& p: \operatorname{E5933000} \\
& \left\{\operatorname{lisp}(a, l)\left(\operatorname{car} v_{1}, v_{2}, v_{3}, v_{4}, v_{5}, v_{6}\right) * \mathrm{pc}(p+4)\right\}
\end{aligned}
$$

The above specification extends the ARM code generator to handle assignments of car $v_{1}$ to s-expression variable $v_{1}$.

$$
\text { rhs ::= let } v_{1}=\operatorname{car} v_{1} \text { in } r h s
$$

\subsection{Stack Usage}

The stack can be used by assignments to and from variables $s_{0}, s_{1}, s_{2}$ etc., e.g. the following let-expressions correspond to machine code which loads register 1 from stack location 3 (three down from top of stack), adds 78 to register 1 and then stores the result in stack location 2 .

$$
\begin{aligned}
& f\left(r_{1}, s_{2}, s_{3}\right)= \text { let } r_{1}=s_{3} \text { in } \\
& \text { let } r_{1}=r_{1}+78 \text { in } \\
& \text { let } s_{2}=r_{1} \text { in } \\
& \quad\left(r_{1}, s_{2}, s_{3}\right)
\end{aligned}
$$

Internally stack accesses are implemented by supplying the decompiler with specifications which specify stack locations using M-assertions (defined formally in [17, informally $\mathrm{M} x y$ asserts that memory location $x$ holds value $y$ ), e.g. the following is the specification used for reading the value of stack location 3 into register 1 . Register 13 is the stack pointer.

$$
\begin{aligned}
& \left\{\mathrm{r} 1 r_{1} * \mathrm{r} 13 s p * \mathrm{M}(s p+12) s_{3} * \mathrm{pc} p\right\} \\
& p: \text { E59D100C } \\
& \left\{\mathrm{r} 1 s_{3} * \mathrm{r} 13 s p * \mathrm{M}(s p+12) s_{3} * \mathrm{pc}(p+4)\right\}
\end{aligned}
$$

The postcondition for the certification theorem proved for the above function $f$ :

$$
\left\{(\mathrm{r} 1, \mathrm{M}(s p+8), \mathrm{M}(s p+12)) \text { is } f\left(r_{1}, s_{2}, s_{3}\right) * \mathrm{r} 13 s p * \mathrm{pc}(p+12)\right\}
$$




\subsection{Subroutines and Procedures}

Subroutines can be in-lined or called as procedures. Each compilation adds a new let-expression into the input languages of the compiler. The added let-expressions describe the compiled code, i.e. they allow subsequent compilations to use the previously compiled code. For example, when the following function (which uses $f$ from above) is compiled, the code for $f$ will be in-lined as in Section 3.1 .

$$
\begin{gathered}
g\left(r_{1}, r_{2}, s_{2}, s_{3}\right)=\text { let }\left(r_{1}, s_{2}, s_{3}\right)=f\left(r_{1}, s_{2}, s_{3}\right) \text { in } \\
\text { let } s_{2}=r_{1} \text { in } \\
\left(r_{1}, r_{2}, s_{2}, s_{3}\right)
\end{gathered}
$$

Note that for simplicity, function calls must match the variable names used when compiling the called function was compiled, e.g. a function compiled as ' $k\left(r_{1}\right)=\ldots$ '. cannot be called as 'let $r_{2}=k\left(r_{2}\right)$ in' since the input is passed to code implementing $k$ in register 1 not in register 2 .

If the compiler had been asked to compile $f$ as a procedure, then the numbering of stack variables needs to be shifted for calls to $f$. Compiling $f$ as a procedure sandwiches the code for $f$ between a push and pop instruction that keep track of the procedure's return address. When $f$ accesses stack locations 2 and 3 (counting in pop-order), these are for caller $g$ locations 1 and 2 .

$$
\begin{gathered}
g\left(r_{1}, r_{2}, s_{1}, s_{2}\right)=\text { let }\left(r_{1}, s_{1}, s_{2}\right)=f\left(r_{1}, s_{1}, s_{2}\right) \text { in } \\
\text { let } s_{2}=r_{1} \text { in } \\
\quad\left(r_{1}, r_{2}, s_{1}, s_{2}\right)
\end{gathered}
$$

\section{Optimising Transformations}

Given a function $f$, the compiler generates code, which it decompiles to produce function $f^{\prime}$ describing the behaviour of the generated code. The code generation phase can perform any optimisations as long as the certification phase can eventually prove $f=f^{\prime}$. In particular, certain instructions can be reordered or removed, and the code's control flow can use special features of the target language.

\subsection{Instruction Reordering}

Instruction reordering is a standard optimisation applied in order to avoid unnecessary pipeline stalls. The compiler presented here supports instruction reordering as is illustrated by the following example. Given a function $f$ which stores $r_{1}$ into stack location $s_{5}$, then loads $r_{2}$ from stack location $s_{6}$, and finally adds $r_{1}$ and $r_{2}$.

$$
\begin{aligned}
& f\left(r_{1}, r_{2}, s_{5}, s_{6}\right)= \text { let } s_{5}=r_{1} \text { in } \\
& \text { let } r_{2}=s_{6} \text { in } \\
& \text { let } r_{1}=r_{1}+r_{2} \text { in } \\
& \quad\left(r_{1}, r_{2}, s_{5}, s_{6}\right)
\end{aligned}
$$


The code corresponding directly to $f$ might cause a pipeline stall as the result of the load instruction (let $r_{2}=s_{6}$ in) may not be available on time for the add instruction (let $r_{1}=r_{1}+r_{2}$ in). It is therefore beneficial to schedule the load instructions as early as possible; the generated code reduces the risk of a pipeline stall by placing the load instruction before the store instruction:

$$
\begin{aligned}
f^{\prime}\left(r_{1}, r_{2}, s_{5}, s_{6}\right)= & \text { let } r_{2}=s_{6} \text { in } \\
& \text { let } s_{5}=r_{1} \text { in } \\
& \text { let } r_{1}=r_{1}+r_{2} \text { in } \\
& \left(r_{1}, r_{2}, s_{5}, s_{6}\right)
\end{aligned}
$$

Valid reorderings of instructions are unnoticeable after expansion of letexpressions, thus the proof of $f=f^{\prime}$ does not need to be smarter to handle this optimisation.

\subsection{Removal of Dead Code}

Live-variable analysis can be applied to the code in order to remove unused or dead code. In the following definition of $f$, the first let-expression is unnecessary.

$$
\begin{aligned}
& f\left(r_{1}, r_{2}, s_{5}, s_{6}\right)= \text { let } r_{1}=s_{5} \text { in } \\
& \text { let } r_{2}=s_{6} \text { in } \\
& \text { let } r_{1}=r_{2}+8 \text { in } \\
& \quad\left(r_{1}, r_{2}, s_{5}, s_{6}\right)
\end{aligned}
$$

The generated code ignores the first let-expression and produces a function $f^{\prime}$ which is, after expansion of let-expressions, identical to $f$.

\subsection{Conditional Execution}

ARM machine code allows conditional execution of nearly all instructions in order to allow short forward jumps to be replaced by conditionally executed instructions (this reduces branch overhead). The compiler produces conditionallyexecuted instruction blocks where short forward jumps would otherwise have been generated. The functions decompiled from conditionally executed instructions are indistinguishable from those decompiled from code with normal jumps (as can be seen in the examples of Section 1 and 4.4).

x86 supports conditional assignment using the conditional-move instruction cmov. For x86, the compiler replaces jumps across register-register moves by conditional-move instructions.

\subsection{Shared Tails}

The compiler's input language supports if-statements that split control, but does not provide direct means for joining control-flow. For example, consider

$$
\text { (if } r 1=0 \text { then } r 2:=23 \text { else } r 2:=56) ; r 1:=4
$$


which can be defined either directly as function $f$ with 'shared tails'

$$
\begin{aligned}
f\left(r_{1}, r_{2}\right)=\text { if } r_{1}=0 & \text { then let } r_{2}=23 \text { in let } r_{1}=4 \text { in }\left(r_{1}, r_{2}\right) \\
& \text { else let } r_{2}=56 \text { in let } r_{1}=4 \text { in }\left(r_{1}, r_{2}\right)
\end{aligned}
$$

or as function $g$ with auxiliary function $g_{2}$ compiled to be in-lined:

$$
\begin{gathered}
g\left(r_{1}, r_{2}\right)=\text { let }\left(r_{1}, r_{2}\right)=g_{2}\left(r_{1}, r_{2}\right) \text { in let } r_{1}=4 \text { in }\left(r_{1}, r_{2}\right) \\
g_{2}\left(r_{1}, r_{2}\right)=\text { if } r_{1}=0 \text { then let } r_{2}=23 \text { in }\left(r_{1}, r_{2}\right) \\
\text { else let } r_{2}=56 \text { in }\left(r_{1}, r_{2}\right)
\end{gathered}
$$

Generating code naively for $f$ would result in two instructions for let $r_{1}=4$ in, one for each branch. The compiler implements an optimisation which detects 'shared tails' so that the code for $f$ will be identical to that produced for $g$. The compiler generates the following ARM code for function $g$ (using conditional execution to avoid inserting short jumps).

$\begin{array}{rll}\text { 0: } & \text { E3510000 } & \text { cmp r1, \#0 } \\ \text { 4: } & 03 A 02017 & \text { moveq } r 2, \# 23 \\ \text { 8: } & 13 A 02038 & \text { movne } r 2, \# 56 \\ \text { 12: } & \text { E3A01004 } & \text { mov r1, \#4 }\end{array}$

\section{Compilation Example: Verified LISP Interpreter}

The following example shows how one can utilise extensions to the input language. A verified interpreter for a LISP-like language is constructed using compilation. Details of the following section will be published as a separate paper.

The LISP interpreter constructed here operates over a simple date-type of s-expressions: Dot $x y$ is a pair, Num $n$ is a number $n$, and Sym $s$ is a symbol $s$, in HOL4, $s$ has type string. Basic operations are defined as follows:

$$
\begin{aligned}
\operatorname{car}(\operatorname{Dot} x y) & =x \\
\operatorname{cdr}(\operatorname{Dot} x y) & =y \\
\operatorname{cons} x y & =\operatorname{Dot} x y \\
\text { plus }(\operatorname{Num} m)(\operatorname{Num} n) & =\operatorname{Num}(m+n) \\
\operatorname{minus}(\operatorname{Num} m)(\operatorname{Num} n) & =\operatorname{Num}(m-n) \\
\operatorname{size}(\operatorname{Num} w) & =0 \\
\operatorname{size}(\operatorname{Sym} s) & =0 \\
\operatorname{size}(\operatorname{Dot} x y) & =1+\text { size } x+\text { size } y
\end{aligned}
$$

A new resource assertion lisp is defined which relates LISP objects to concrete memory representations: lisp $(a, l)\left(v_{1}, v_{2}, v_{3}, v_{4}, v_{5}, v_{6}\right)$ states that a heap is located at address $a$, has capacity $l$, and that s-expressions $v_{1}, v_{2}, v_{3}, v_{4}, v_{5}, v_{6}$ are stored in this heap. The definition of lisp is omitted in this presentation. 
Machine code for basic operations has been proved (in various ways using decompilation and compilation) to implement basic assertions, e.g. ARM code for storing car $v_{1}$ into $v_{1}$ :

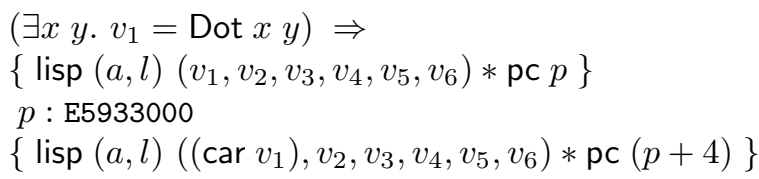

A memory allocator with a built-in copying garbage collector (a Cheney garbage collector [4]) is used to implement creation of a new pair Dot $v_{1} v_{2}$. The precondition of this operation requires the heap to have enough space to accommodate a new cons-cell.

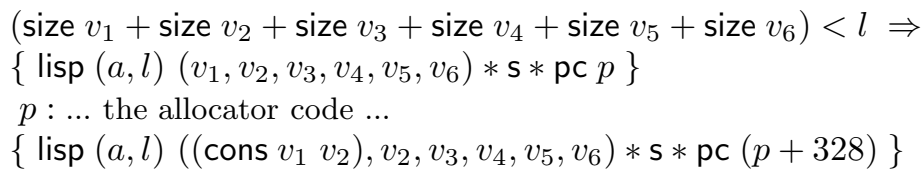

When the above specifications are supplied to the compiler it knows what machine code to generate for two new commands: one for calculating car of $v_{1}$ and one for storing cons $v_{1} v_{2}$ into $v_{1}$ :

$$
\text { let } v_{1}=\operatorname{car} v_{1} \text { in } \quad \text { let } v_{1}=\text { cons } v_{1} v_{2} \text { in }
$$

Once the compilers language had been extended with sufficiently many such primitive operations, a LISP interpreter was compiled using our proof-producing compiler. The top-level specification function defining a simple LISP interpreter lisp_eval is listed in Figure1. When lisp_eval is compiled, code is generated and a theorem is proved which state that this LISP interpreter is implemented by the generated machine code, in this case ARM code.

$$
\begin{aligned}
& \text { lisp_eval_pre }\left(v_{1}, v_{2}, v_{3}, v_{4}, v_{5}, v_{6}, l\right) \Rightarrow \\
& \left\{\operatorname{lisp}(a, l)\left(v_{1}, v_{2}, v_{3}, v_{4}, v_{5}, v_{6}\right) * \mathrm{~s} * \mathrm{pc} p\right\} \\
& p: \ldots \text { the generated code } \ldots \\
& \left\{\operatorname{lisp}(a, l)\left(\text { lisp_eval }\left(v_{1}, v_{2}, v_{3}, v_{4}, v_{5}, v_{6}, l\right)\right) * \mathrm{~s} * \mathrm{pc}(p+3012)\right\}
\end{aligned}
$$

Here lisp_eval_pre has collected the various side-conditions that need to be true for proper execution of the code.

\section{Summary and Discussion of Related Work}

This paper has described how an extensible proof-producing compiler can be implemented using decompilation into logic [18]. The implementation required only a light-weight certification phase (approximately 100 lines of ML code) to be programmed, but still proves functional equivalence between the source and target programs. In contrast to previous work 812 1314, correctness proofs are here separated from code generation. 


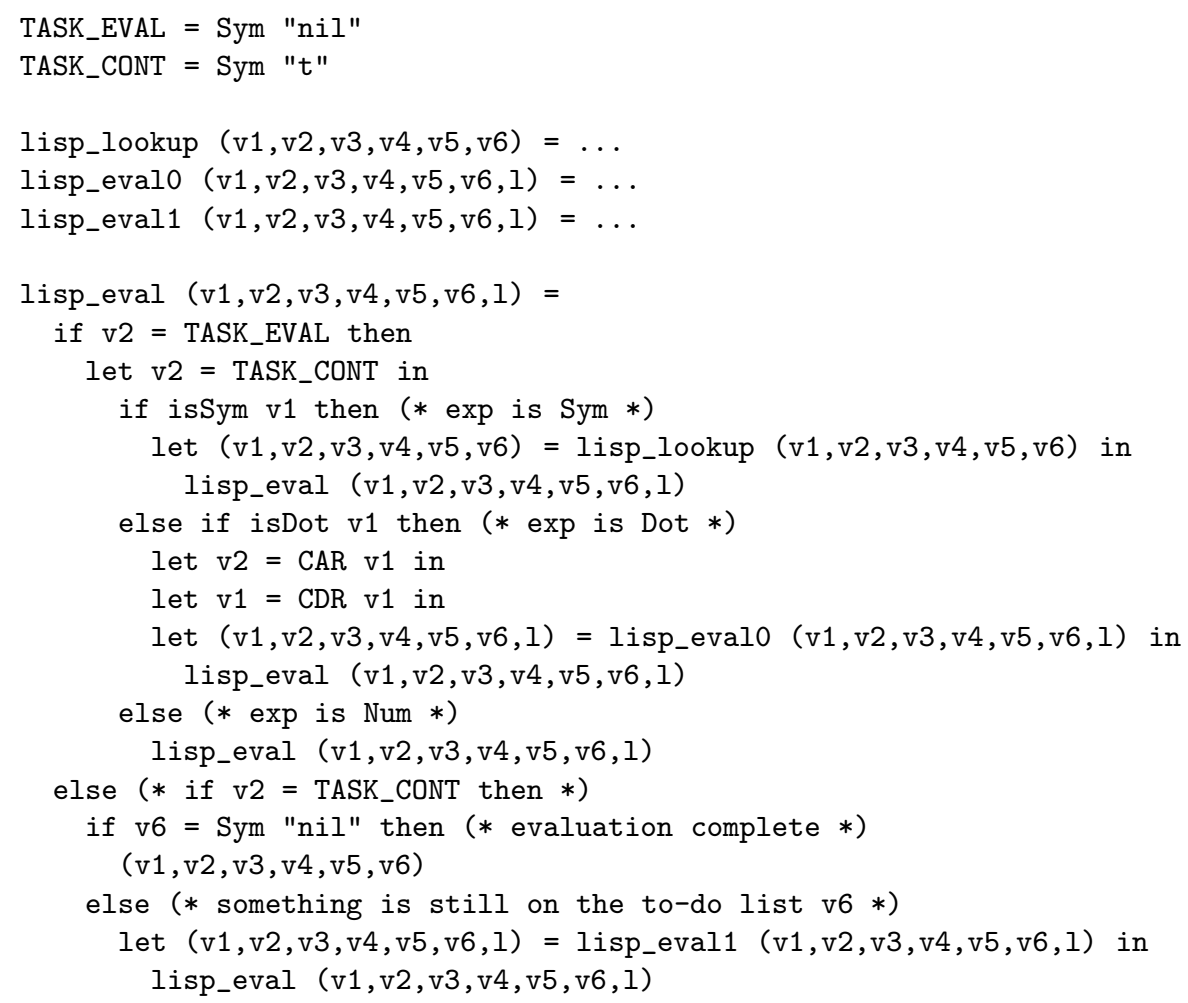

Fig. 1. The top-level definition of lisp_eval in HOL4

For each run, the compiler generates code and then proves that the code is correct. This is an idea for which Pnueli et al. 20] coined the term translation validation. There are two basic approaches to translation validation: (1) code generation is instrumented to generate proofs, and (2) code generation proceeds as usual then the certification phase attempts to guess the proofs. Approach 1 is generally considered more feasible [21]. However, Necula [19] showed that approach 2 is feasible even for aggressively optimising compilers such as GNU gcc [2]. Necula built into his certification phase heuristics that attempt to guess which optimisations were performed. The compiler presented here also implements approach 2 , but restricts the (initial) input language and the optimisations to such an extent that the certification phase does not need any guesswork.

An alternative to producing a proof for each run is to prove the compiler correct. A recent, particularly impressive, milestone in compiler verification was achieved by Leroy [11, who proved the correctness of an optimising compiler which takes a significant subset of $\mathrm{C}$ as input and produces PowerPC assembly $\operatorname{cod} 2$ as output. As part of this project Tristan and Leroy 22] verified multiple

${ }^{2}$ The work presented here builds on Leroy's specification of PowerPC assembly code. 
translation validators. Other recent work is [10 15 1135]. We chose not to verify our compiler/translation validator, since our compiler constructs all of its proofs in the HOL4 theorem prover. The trusted computing base (TCB) of our compiler is HOL4 and the specifications of the target machine languages. It seems that the user-defined extensions such as those in the LISP example would have been much harder to implement in a verified compiler, since verifying a compiler involves defining a deep embedding of the input language.

The VLISP project 9], which produced verified on-paper proofs for an implementation of a larger subset of LISP, is related to the example above of constructing a verified LISP interpreter. The fact that the proof presented here is mechanised and goes down to detailed models of commercial machine languages distinguishes this work from the VLISP project which stopped at the level of verified algorithms.

Acknowledgements. We thank Anthony Fox, Xavier Leroy and Susmit Sarkar for allowing us to use their processor models for this work. We also thank Thomas Tuerk, Aaron Coble and the anonymous reviewers for comments on earlier drafts. The first author is grateful for funding from EPSRC, UK.

\section{References}

1. The Netwide Assembler, http://www.nasm.us/

2. The GNU Project. GCC, the GNU Compiler Collection, http://gcc.gnu.org/

3. Benton, N., Zarfaty, U.: Formalizing and verifying semantic type soundness of a simple compiler. In: Leuschel, M., Podelski, A. (eds.) Principles and Practice of Declarative Programming (PPDP), pp. 1-12. ACM, New York (2007)

4. Cheney, C.J.: A non-recursive list compacting algorithm. Commun. ACM 13(11), 677-678 (1970)

5. Chlipala, A.J.: A certified type-preserving compiler from lambda calculus to assembly language. In: Programming Language Design and Implementation (PLDI), pp. 54-65. ACM, New York (2007)

6. Crary, K., Sarkar, S.: Foundational certified code in a metalogical framework. Technical Report CMU-CS-03-108, Carnegie Mellon University (2003)

7. Fox, A.: Formal specification and verification of ARM6. In: Basin, D., Wolff, B. (eds.) TPHOLs 2003. LNCS, vol. 2758, pp. 25-40. Springer, Heidelberg (2003)

8. Gordon, M., Iyoda, J., Owens, S., Slind, K.: Automatic formal synthesis of hardware from higher order logic. Electr. Notes Theor. Comput. Sci. 145, 27-43 (2006)

9. Guttman, J., Ramsdell, J., Wand, M.: VLISP: A verified implementation of scheme. Lisp and Symbolic Computation 8(1/2), 5-32 (1995)

10. Klein, G., Nipkow, T.: A machine-checked model for a Java-like language, virtual machine, and compiler. ACM Trans. Program. Lang. Syst. 28(4), 619-695 (2006)

11. Leroy, X.: Formal certification of a compiler back-end, or: programming a compiler with a proof assistant. In: Principles of Programming Languages (POPL), pp. 42 54. ACM Press, New York (2006)

12. Li, G.-D., Owens, S., Slind, K.: Structure of a proof-producing compiler for a subset of higher order logic. In: De Nicola, R. (ed.) ESOP 2007. LNCS, vol. 4421, pp. 205-219. Springer, Heidelberg (2007) 
13. Li, G.-D., Slind, K.: Compilation as rewriting in higher order logic. In: Pfenning, F. (ed.) CADE 2007. LNCS, vol. 4603, pp. 19-34. Springer, Heidelberg (2007)

14. Li, G., Slind, K.: Trusted source translation of a total function language. In: Ramakrishnan, C.R., Rehof, J. (eds.) TACAS 2008. LNCS, vol. 4963, pp. 471-485. Springer, Heidelberg (2008)

15. Meyer, T., Wolff, B.: Tactic-based optimized compilation of functional programs. In: Filliâtre, J.-C., Paulin-Mohring, C., Werner, B. (eds.) TYPES 2004. LNCS, vol. 3839, pp. 201-214. Springer, Heidelberg (2006)

16. Myreen, M.O., Fox, A.C.J., Gordon, M.J.C.: A Hoare logic for ARM machine code. In: Arbab, F., Sirjani, M. (eds.) FSEN 2007. LNCS, vol. 4767, pp. 272-286. Springer, Heidelberg (2007)

17. Myreen, M.O., Gordon, M.J.C.: A Hoare logic for realistically modelled machine code. In: Grumberg, O., Huth, M. (eds.) TACAS 2007. LNCS, vol. 4424, pp. 568 582. Springer, Heidelberg (2007)

18. Myreen, M.O., Slind, K., Gordon, M.J.C.: Machine-code verification for multiple architectures - An application of decompilation into logic. In: Formal Methods in Computer Aided Design (FMCAD). IEEE, Los Alamitos (2008)

19. Necula, G.C.: Translation validation for an optimizing compiler. In: Programming Language Design and Implementation (PLDI), pp. 83-94. ACM, New York (2000)

20. Pnueli, A., Siegel, M., Singerman, E.: Translation validation. In: Steffen, B. (ed.) TACAS 1998. LNCS, vol. 1384, pp. 151-166. Springer, Heidelberg (1998)

21. Rinard, M.C.: Credible compilation. In: Jähnichen, S. (ed.) CC 1999. LNCS, vol. 1575. Springer, Heidelberg (1999)

22. Tristan, J.-B., Leroy, X.: Formal verification of translation validators: a case study on instruction scheduling optimizations. In: Principles of Programming Languages (POPL), pp. 17-27. ACM, New York (2008) 